\title{
Simulation of ferrofluids by BEM
}

\author{
J. Ravnik \& L. Škerget \\ Faculty of Mechanical Engineering, University of Maribor, Slovenia
}

\begin{abstract}
In this paper the Boundary element method (BEM) was applied to simulate viscous flow of ferrofluids under the influence of a homogenous external magnetic field. We present a derivation of the boundary integral form of the vorticity transport equation, which includes magnetic body force terms. Furthermore, discretization and numerical solution of the coupled flow and heat transfer problems are presented.

The derived numerical model has been used to study the effect of a homogenous external magnetic field on the natural convection of ferrofluids in a differentially heated enclosure. We established that the effects are negligible for magnetic Rayleigh numbers less or equal to the flow Rayleigh number. On the other hand, the magnetic body force changes the flow field significantly and contributes to the instability of the flow.

Keywords: boundary element method, velocity-vorticity formulation, ferrofluid, natural convection, magnetic field.
\end{abstract}

\section{Introduction}

A ferrofluid is a colloidal suspension of magnetic nanoparticles. The flow field of these fluids can be significantly altered by the application of magnetic fields. They can be used to develop microscale heat exchangers in MEMS devices. Since in miniature devices the flow is limited to low Reynolds numbers the heat is transferred mainly by diffusion, the magnetic field can be used to manipulate and enhance convection.

Recently many researches have studied ferrofluids under the influence of the magnetic field (Hong et al. [4], Lajvardi et al. [5], Tynjälä et al. [13]) for a wide variety of applications. In this work we derive a boundary-domain integral form of the vorticity transport equation for a ferrofluid and present its numerical solution. 
Thermal convection of a ferrofluid under a homogenous external magnetic field is investigated as the model problem.

\section{Governing equations}

The magnetic field in the ferrofluid conforms to the Maxwell's equations in static form as

$$
\vec{\nabla} \cdot \vec{B}=0, \quad \vec{\nabla} \times \vec{H}=0,
$$

where $\vec{B}$ is the magnetic field density and $\vec{H}$ is the magnetic field strength. They are related by

$$
\vec{B}=\mu_{0}\left(1+\chi_{m}\right) \vec{H}
$$

where $\chi_{m}$ is the magnetic susceptibility of the ferrofluid and vacuum permeability $\mu_{0}=4 \pi \cdot 10^{-7} \mathrm{~N} / A^{2}$. The magnetic field causes magnetization of the ferrofluid. For small variations of temperature, which will be the subject of this investigation, the change of magnetization of the ferrofluid $d M=-(\partial M / \partial T)_{H} d H+$ $(\partial M / \partial H)_{T} d T$ can be written in linearized form as

$$
M-M^{\star}=-K\left(T-T_{0}\right)+\chi_{r}\left(H-H^{\star}\right),
$$

where $K=-(\partial M / \partial T)_{H}$ is the pyromagnetic coefficient, $\chi_{r}=(\partial M / \partial H)_{T}$ is the differential magnetic susceptibility of the fluid (Ganguly et al. [2]), $M^{\star}$ and $H^{\star}$ are the equilibrium values around which linearization is preformed and $T_{0}$ is the reference temperature. The pyromagnetic coefficient depends on the thermal disorientating motion of magnetic nanoparticles, thermal dependence of magnetic moment, $m_{p}$ of particles and thermal expansion of the fluid. The first effect does not contribute in the case of single domain magnetic nanoparticles, thus (Berkovsky et al. [1]) pyromagnetic coefficient can be expressed as

$$
K=M\left(\beta_{m}+\beta\right),
$$

where $\beta=-(1 / \rho)(\partial \rho / \partial T)$ is the thermal expansion of the fluid and $\beta_{m}=$ $-(1 / \rho)\left(\partial m_{p} / \partial T\right)$ is the relative temperature coefficient of the magnetic moment of a single particle. For most ferrofluids it is valid that $\beta \gg \beta_{m}$ for temperatures below the Courie point (Müller and Engel [7]). Thus, by neglecting $\beta_{m}$, we rewrite equation (3) as

$$
M=M^{\star}-M \beta\left(T-T_{0}\right)+\chi_{r}\left(H-H^{\star}\right)
$$

Taking into account that the fluid does not possess any residual magnetization when there is no magnetizing force, $M^{\star}=0, H^{\star}=0$ and assuming that the differential magnetic susceptibility is constant over the range of $H$ considered, $\chi_{r}=\chi_{0}$, we have

$$
\frac{M}{H}=\chi_{m}=\frac{\chi_{0}}{1+\beta\left(T-T_{0}\right)},
$$

where $\chi_{m}$ is the total magnetic susceptibility, which forms an equation of state between magnetization and magnetic field strength, $\vec{M}=\chi_{m} \vec{H}$. In this setting the magnetic susceptibility is a function of temperature only. 
In this paper we assume an incompressible viscous Newtonian fluid with constant material properties: density $\rho$, viscosity $\nu$, specific heat capacity $c_{p}$ and thermal conductivity $k$. The continuity equation (mass conservation law) within this approximation

$$
\vec{\nabla} \cdot \vec{v}=0
$$

requires the velocity field $\vec{v}$ to be solenoidal, i.e. divergence free. In order to write the Navier-Stokes equations buoyancy is modelled within the Boussinesq approximation. Density variations with temperature $\rho(T)=\rho\left[1-\beta\left(T-T_{0}\right)\right]$ are considered only in the buoyancy term and defined by the thermal volume expansion coefficient $\beta$ and the temperature difference. The momentum transport equation reads for a couple flow and heat transfer problem of a ferrofluid in a magnetic field as

$$
\frac{\partial \vec{v}}{\partial t}+(\vec{v} \cdot \vec{\nabla}) \vec{v}=-\frac{1}{\rho} \vec{\nabla} p+\nu \nabla^{2} \vec{v}-\beta\left(T-T_{0}\right) \vec{g}+\frac{1}{\rho}(\vec{M} \cdot \vec{\nabla}) \vec{B},
$$

where the last term in the equation is the Kelvin body force per unit mass $\vec{f}_{K}=\frac{1}{\rho}(\vec{M} \cdot \vec{\nabla}) \vec{B}$. Using equations (1) and (2) we rewrite the Kelvin body force as

$$
\vec{f}_{K}=\frac{\mu_{0} \chi_{m}}{2 \rho}\left(1+\chi_{m}\right) \vec{\nabla}(\vec{H} \cdot \vec{H})+\frac{\mu_{0} \chi_{m}}{\rho} \vec{H}\left(\vec{H} \cdot \vec{\nabla} \chi_{m}\right) .
$$

Since the magnetic susceptibility depends solely on temperature, $\chi_{m}=\chi_{m}(T)$, we can relate its gradient with the temperature gradient by making use of equation (6):

$$
\vec{\nabla} \chi_{m}=\frac{\beta \chi_{0}}{\left(1+\beta\left(T-T_{0}\right)\right)^{2}} \vec{\nabla} T .
$$

Using (6) and (10) the Kelvin body force in equation (9) can be rewritten as

$$
\vec{f}_{K}=\frac{\mu_{0} \chi_{0} \beta}{\rho}\left(\frac{1}{2} \frac{\frac{1+\chi_{0}}{\beta}+\left(T-T_{0}\right)}{\left(1+\beta\left(T-T_{0}\right)\right)^{2}} \vec{\nabla} H^{2}+\frac{\chi_{0}}{\left(1+\beta\left(T-T_{0}\right)\right)^{3}} \vec{H}(\vec{H} \cdot \vec{\nabla} T)\right) .
$$

The Kelvin body force has two terms. The first vanishes if the magnetic field is uniform, i.e. if there are no magnetic field gradients. The second vanishes, if the ferrofluid temperature is uniform, i.e. if there are no temperature gradients.

Since we are solving a coupled momentum - heat transport problem, the field functions were non-dimensionalized in the following manner: $\vec{v} \rightarrow \frac{\vec{v}}{v_{0}}, \vec{g} \rightarrow \frac{\vec{g}}{g_{0}}$, $\vec{r} \rightarrow \frac{\vec{r}}{L}, \vec{\omega} \rightarrow \frac{\vec{\omega} L}{v_{0}}, t \rightarrow \frac{v_{0} t}{L}, T \rightarrow \frac{T-T_{0}}{\Delta T}, p \rightarrow \frac{p}{p_{0}}, v_{0}=\frac{k}{\rho c_{p} L}, H \rightarrow \frac{H}{H_{0}}$, where $p$ is pressure, $L$ is the characteristic length scale (height of the hot and cold walls in our model problem) and $\vec{\omega}$ is vorticity. With the above stated assumptions the momentum transport equation (momentum conservation law) in non-dimensional 
form reads as:

$$
\begin{gathered}
\frac{\partial \vec{v}}{\partial t}+(\vec{v} \cdot \vec{\nabla}) \vec{v}=-\operatorname{Pr} \operatorname{Ra} T \vec{g}-\frac{1}{E u} \vec{\nabla} p+\operatorname{Pr} \nabla^{2} \vec{v}+\operatorname{Pr} R a_{m} \vec{f}_{K}^{\prime}, \\
\vec{f}_{K}^{\prime}=\frac{1}{2} \frac{\frac{1+\chi_{0}}{\beta \Delta T}+T}{(1+T \beta \Delta T)^{2}} \vec{\nabla}(\vec{H} \cdot \vec{H})+\frac{\chi_{0}}{(1+T \beta \Delta T)^{3}} \vec{H}(\vec{H} \cdot \vec{\nabla} T),
\end{gathered}
$$

with the Prandtl $\operatorname{Pr}$, enclosure height based Rayleigh $R a$, magnetic Rayleigh $R a_{m}$ (Ganguly et al. [3]) and Euler Eu numbers defined as:

$$
\operatorname{Pr}=\frac{\nu \rho c_{p}}{k}, \quad R a=\frac{g_{0} \beta \Delta T L^{3}}{\nu \frac{k}{\rho c_{p}}}, \quad R a_{m}=\frac{\mu_{0} \chi_{0} H_{0}^{2} L^{2} \beta \Delta T}{\rho \nu \frac{k}{\rho c_{p}}}, \quad E u=\frac{\rho_{0} v_{0}^{2}}{p_{0}} .
$$

Furthermore, we assume that no internal energy sources are present in the fluid. We will not deal with high velocity flow of highly viscous fluid, hence we will neglect irreversible viscous dissipation. With this, the internal energy conservation law, written with temperature as the unknown variable, reads as:

$$
\frac{\partial T}{\partial t}+(\vec{v} \cdot \vec{\nabla}) T=\nabla^{2} T
$$

\subsection{Velocity-vorticity formulation}

The mass conservation law (7) and momentum transport equation (12) are rewritten in velocity-vorticity form by introducing vorticity $\vec{\omega}$. Vorticity is defined by the curl of velocity, $\vec{\omega}=\vec{\nabla} \times \vec{v}$. After taking a curl of the governing equations, we obtain

$$
\nabla^{2} \vec{v}+\vec{\nabla} \times \vec{\omega}=0
$$

the kinematics equation, which relates the velocity and vorticity fields for every point in space and time. Both, the vorticity and velocity fields must be solenoidal, in order for this equation to be fulfilled.

The final form of the vorticity transport equation reads as

$$
\frac{\partial \vec{\omega}}{\partial t}+(\vec{v} \cdot \vec{\nabla}) \vec{\omega}=(\vec{\omega} \cdot \vec{\nabla}) \vec{v}+\operatorname{Pr} \nabla^{2} \vec{\omega}-\operatorname{Pr} R a \vec{\nabla} \times T \vec{g}+\operatorname{Pr} R a_{m} \vec{\nabla} \times \vec{f}_{K}^{\prime} .
$$

Equation (16) equates the Stokes rate of change of vorticity on the left hand side with the vortex twisting and stretching term, the diffusion term, buoyancy and Kelvin body force term on the right hand side.

\section{BEM solution of the transport equation}

\subsection{Boundary-domain integral formulation}

Let us consider a domain $\Omega$ with a position vector $\vec{r} \in \mathbb{R}^{3}$. The boundary of the domain is $\Gamma=\partial \Omega$. In this work we are simulating a natural convection 
phenomena up to $R a \leqslant 10^{6}$. The flow field in this case is steady, thus we may write $\partial \vec{\omega} / \partial t=0$. The boundary-domain integral form of the steady vorticity transport equation (16) is

$$
\begin{array}{r}
c(\vec{\xi}) \vec{\omega}(\vec{\xi})+\int_{\Gamma} \vec{\omega} \vec{\nabla} u^{\star} \cdot \vec{n} d \Gamma=\int_{\Gamma} u^{\star} \vec{q} d \Gamma \\
+\frac{1}{\operatorname{Pr}} \int_{\Omega} u^{\star}\{(\vec{v} \cdot \vec{\nabla}) \vec{\omega}-(\vec{\omega} \cdot \vec{\nabla}) \vec{v}\} d \Omega \\
+R a \int_{\Omega} u^{\star} \vec{\nabla} \times T \vec{g} d \Omega-R a_{m} \int_{\Omega} u^{\star} \vec{\nabla} \times \vec{f}_{K}^{\prime} d \Omega,
\end{array}
$$

where $\vec{\xi}$ is the source or collocation point, $\vec{n}$ is a vector normal to the boundary, pointing out of the domain and $u^{\star}$ is the fundamental solution for the diffusion operator:

$$
u^{\star}=\frac{1}{4 \pi|\vec{\xi}-\vec{r}|} .
$$

$c(\vec{\xi})$ is the geometric factor defined as $c(\vec{\xi})=\alpha / 4 \pi$, where $\alpha$ is the inner angle with origin in $\vec{\xi}$. If $\vec{\xi}$ lies inside of the domain then $c(\vec{\xi})=1 ; c(\vec{\xi})=1 / 2$, if $\vec{\xi}$ lies on a smooth boundary. Vorticity on the boundary $\vec{\omega}(\vec{r})$ or vorticity flux on the boundary $\vec{q}(\vec{r})=\vec{\nabla} \vec{\omega}(\vec{r}) \cdot \vec{n}$ are prescribed as boundary conditions.

All domain integrals on the right hand side of equation (17) include derivatives of the unknown field functions. Škerget and Alujevič [12] and Škerget et al. [11] has shown how to use algebraic relations to move the derivative from the velocity and vorticity field functions. Body force integrals (buoyancy and Kelvin force) are both of the same nature and can be thus handled in similar manner. Let $\vec{f}=R a T \vec{g}-R a_{m} \vec{f}_{K}^{\prime}$ then we need to calculate the following integral

$$
\int_{\Omega} u^{\star} \vec{\nabla} \times \vec{f} d \Omega .
$$

The kernel of this integral is the curl of sum of the Kelvin and the buoyancy forces multiplied by the fundamental solution. In order to avoid calculation of the curl, we use a vector relation $u^{\star} \vec{\nabla} \times \vec{f}=\vec{\nabla} \times\left(u^{\star} \vec{f}\right)+\vec{f} \times \vec{\nabla} u^{\star}$ and obtain

$$
\int_{\Omega} u^{\star} \vec{\nabla} \times \vec{f} d \Omega=\int_{\Omega} \vec{\nabla} \times\left(u^{\star} \vec{f}\right) d \Omega+\int_{\Omega} \vec{f} \times \vec{\nabla} u^{\star} d \Omega .
$$

The first integral on the right hand side of (20) can be transformed into a boundary integral by the Gauss clause, yielding

$$
\int_{\Omega} u^{\star} \vec{\nabla} \times \vec{f} d \Omega=-\int_{\Gamma} u^{\star} \vec{f} \times \vec{n} d \Gamma+\int_{\Omega} \vec{f} \times \vec{\nabla} u^{\star} d \Omega .
$$

Taking into account (21) and the results of Ravnik et al. [9] the integral form of the vorticity transport equation without derivatives of field functions, for the $j$-th 
component of vorticity, is

$$
\begin{array}{r}
c(\vec{\xi}) \omega_{j}(\vec{\xi})+\int_{\Gamma} \omega_{j} \vec{\nabla} u^{*} \cdot \vec{n} d \Gamma=\int_{\Gamma} u^{*} q_{j} d \Gamma \\
+\frac{1}{P r} \int_{\Gamma} \vec{n} \cdot\left\{u^{*}\left(\vec{v} \omega_{j}-\vec{\omega} v_{j}\right)\right\} d \Gamma-\frac{1}{P r} \int_{\Omega}\left(\vec{v} \omega_{j}-\vec{\omega} v_{j}\right) \cdot \vec{\nabla} u^{*} d \Omega \\
-\int_{\Gamma}\left(u^{\star} \vec{f} \times \vec{n}\right)_{j} d \Gamma+\int_{\Omega}\left(\vec{f} \times \vec{\nabla} u^{\star}\right)_{j} d \Omega .
\end{array}
$$

\subsection{Sub-domain discretization technique}

In the subdomain BEM method we make a mesh of the entire domain $\Omega$ and name each mesh element a subdomain. Equation (22) is written for each of the subdomains. In order to obtain a discrete version of (22) we use shape functions to interpolate field functions and flux across the boundary and inside of the subdomain. In this work we used hexahedral subdomains, which enable continuous quadratic interpolation of field functions. The boundary of each hexahedron consists of 6 boundary elements. On each boundary element we interpolate the flux using discontinuous linear interpolation scheme. A function is interpolated over a boundary elements as $T=\sum \varphi_{i} T_{i}$, inside each subdomain as $T=\sum \Phi_{i} T_{i}$, while flux is interpolated over boundary elements as $q=\sum \phi_{i} q_{i}$. The following integrals must be calculated:

$$
\begin{aligned}
& {[H]=\int_{\Gamma} \varphi_{i} \vec{\nabla} u^{\star} \cdot \vec{n} d \Gamma, \quad[G]=\int_{\Gamma} \phi_{i} u^{\star} d \Gamma,} \\
& {[\vec{A}]=\int_{\Gamma} \varphi_{i} \vec{n} u^{\star} d \Gamma, \quad[\vec{D}]=\int_{\Omega} \Phi_{i} \vec{\nabla} u^{\star} d \Omega .}
\end{aligned}
$$

The square brackets denote integral matrices. Each source point location yields one row in these matrices. In order to calculate the integrals, a Gaussian quadrature algorithm is used. The integrals are calculated in local coordinate system via weighted summation of up to 48 integration points per coordinate axis. Calculation of the free coefficient $c(\vec{\xi})$ is preformed indirectly. If we consider a rigid body movement, $u=1, q=0$, we see that the sum of all $[H]$ matrix elements for one source point must be equal to 0 , thus we may use this fact to calculate $c(\vec{\xi})$. The calculated $c(\vec{\xi})$ are added to the diagonal terms of the $[H]$ matrix.

The source point is set to all function and flux nodes in each subdomain. By letting curly brackets denote vectors of nodal values of field functions, we may write the discrete vorticity transport equation in component form as:

$$
\begin{array}{r}
{[H]\left\{\omega_{x}\right\}=\frac{1}{P r}\left[A_{y}\right]\left\{v_{y} \omega_{x}-\omega_{y} v_{x}\right\}+\frac{1}{P r}\left[A_{z}\right]\left\{v_{z} \omega_{x}-\omega_{z} v_{x}\right\}} \\
-\frac{1}{P r}\left[D_{y}\right]\left\{v_{y} \omega_{x}-\omega_{y} v_{x}\right\}-\frac{1}{P r}\left[D_{z}\right]\left\{v_{z} \omega_{x}-\omega_{z} v_{x}\right\} \\
+[G]\left\{q_{x}\right\}+\left[A_{y}\right]\left\{f_{z}\right\}-\left[A_{z}\right]\left\{f_{y}\right\}-\left[D_{y}\right]\left\{f_{z}\right\}+\left[D_{z}\right]\left\{f_{y}\right\},
\end{array}
$$




$$
\begin{array}{r}
{[H]\left\{\omega_{y}\right\}=\frac{1}{\operatorname{Pr}}\left[A_{x}\right]\left\{v_{x} \omega_{y}-\omega_{x} v_{y}\right\}+\frac{1}{\operatorname{Pr}}\left[A_{z}\right]\left\{v_{z} \omega_{y}-\omega_{z} v_{y}\right\}} \\
-\frac{1}{\operatorname{Pr}}\left[D_{x}\right]\left\{v_{x} \omega_{y}-\omega_{x} v_{y}\right\}-\frac{1}{\operatorname{Pr}}\left[D_{z}\right]\left\{v_{z} \omega_{y}-\omega_{z} v_{y}\right\} \\
+[G]\left\{q_{y}\right\}+\left[A_{z}\right]\left\{f_{x}\right\}-\left[A_{x}\right]\left\{f_{z}\right\}+\left[D_{x}\right]\left\{f_{z}\right\}-\left[D_{z}\right]\left\{f_{x}\right\}, \\
{[H]\left\{\omega_{z}\right\}=\frac{1}{\operatorname{Pr}}\left[A_{x}\right]\left\{v_{x} \omega_{z}-\omega_{x} v_{z}\right\}+\frac{1}{\operatorname{Pr}}\left[A_{y}\right]\left\{v_{y} \omega_{z}-\omega_{y} v_{z}\right\}} \\
-\frac{1}{\operatorname{Pr}}\left[D_{x}\right]\left\{v_{x} \omega_{z}-\omega_{x} v_{z}\right\}-\frac{1}{\operatorname{Pr}}\left[D_{y}\right]\left\{v_{y} \omega_{z}-\omega_{y} v_{z}\right\} \\
+[G]\left\{q_{z}\right\}+\left[A_{x}\right]\left\{f_{y}\right\}-\left[A_{y}\right]\left\{f_{x}\right\}-\left[D_{x}\right]\left\{f_{y}\right\}+\left[D_{y}\right]\left\{f_{x}\right\} .
\end{array}
$$

Since neighbouring subdomains share nodes, the systems of linear equations (25), (26) and (27) are over-determined. After taking into account the boundary conditions, we solve them using a least squares solver (Paige and Saunders [8]). All integrals depend only on the shape of subdomains and as such may be calculated only once, prior to the start of the nonlinear iterative process.

\section{Nonlinear solution algorithm}

The system of equations (15), (16) and (14) is solved in a nonlinear loop of three steps. First, the boundary vorticity values are calculated by solving the kinematics equation with the help of a single domain BEM. Second, the calculation of the domain velocity values is achieved by solving the kinematics equation with a subdomain BEM. Next the energy equation is solved by subdomain BEM and finally, the vorticity transport equation for domain vorticity values using the boundary values from the solution of the kinematics equation is solved by a subdomain BEM. Solution of the vorticity equation is described above, details of the solution of the other equations are given by Ravnik et al. [10].

\section{Numerical tests}

We have simulated a ferrofluid with the following representative properties: $\chi_{0}=0.1, \beta=5.6 \cdot 10^{-4} \mathrm{~K}^{-1}, \rho=1180 \mathrm{~kg} / \mathrm{m}^{3}, \nu=5.93 \cdot 10^{-6} \mathrm{~m}^{2} / \mathrm{s}, \alpha=$ $1.19 \cdot 10^{-7} \mathrm{~m} / \mathrm{s}^{2}$ and $\operatorname{Pr}=49.8[3,6]$. We consider the ferrofluid to be enclosed in a cubical $(L \times L \times L)$ enclosure and subjected to a temperature gradient on two vertical walls. All other wall are adiabatic. No-slip velocity boundary conditions are applied on all walls. The problem setup is shown in Figure 1. In such differentially heated enclosure fluid due to buoyancy forces flows up the hot wall and down the cold wall creating a vortex in the enclosure and leading to temperature stratification within the enclosure. In this work we investigate the behaviour of a ferrofluid in such an enclosure subjected to external magnetic fields.

We consider the enclosure placed in a homogenous external magnetic field. The magnetic field is aligned with the enclosure walls and has the direction form the hot 


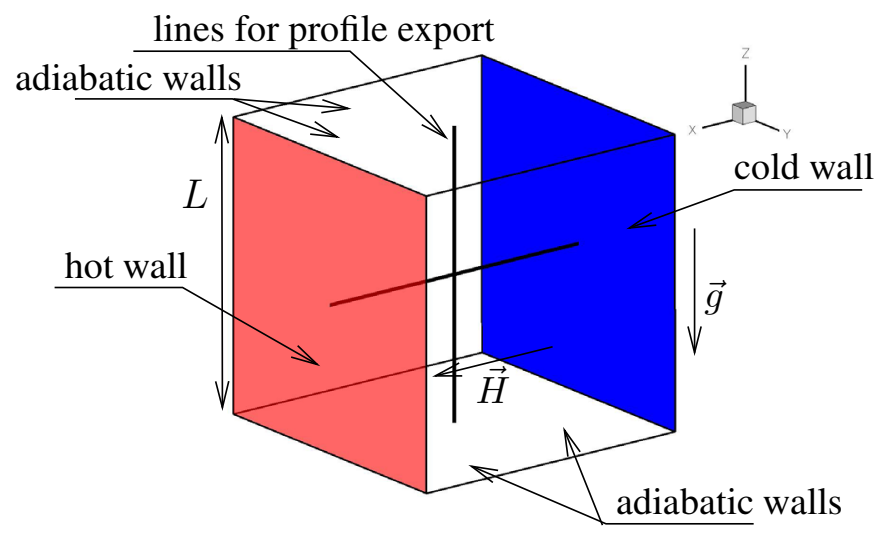

Figure 1: Problem setup and boundary conditions. No-slip velocity boundary conditions are applied on all walls.

to the cold wall. We consider flow of Rayleigh number for $R a=10^{4}$ to $R a=10^{6}$ and magnetic Rayleigh number values from $R a=10^{4}$ to $R a=10^{6}$.

\subsection{Results}

Figure 2 shows the temperature contours at $R a=10^{4}$ within the enclosure for various magnetic fields. We observe that the overall structure of the flow and temperature fields remain the same regardless of the magnetic field. Temperature stratification in the centre of the enclosure is evident in all cases as well as the existence of the primary vortex.

In order to capture the effect of the magnetic field flow and temperature field was investigated on two profiles, which run through the centre of the enclosure. The location of the profiles is shown in Figure 1. Figures 3-8 show temperature, $x$ velocity component and $y$ vorticity component on both profiles for all simulated cases.
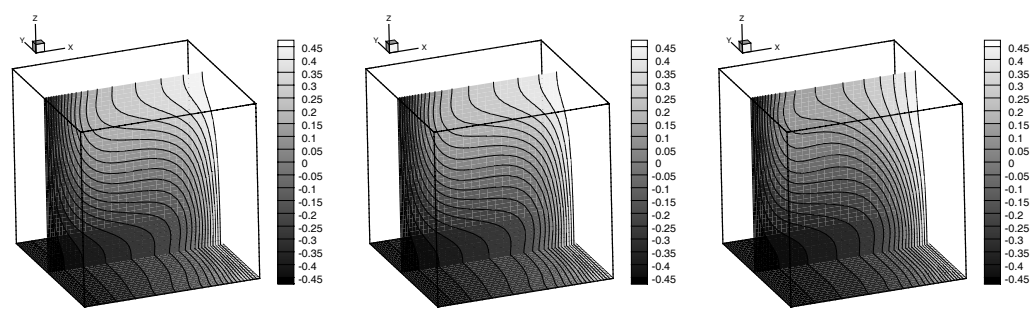

Figure 2: Temperature contours in the enclosure, $R a=10^{4}$. No magnetic field (left), homogenous external magnetic field $\vec{H}=\left(H_{0}, 0,0\right)$ yielding $R a_{m}=10^{4}$ (center) and $R a_{m}=10^{5}$ (right). 

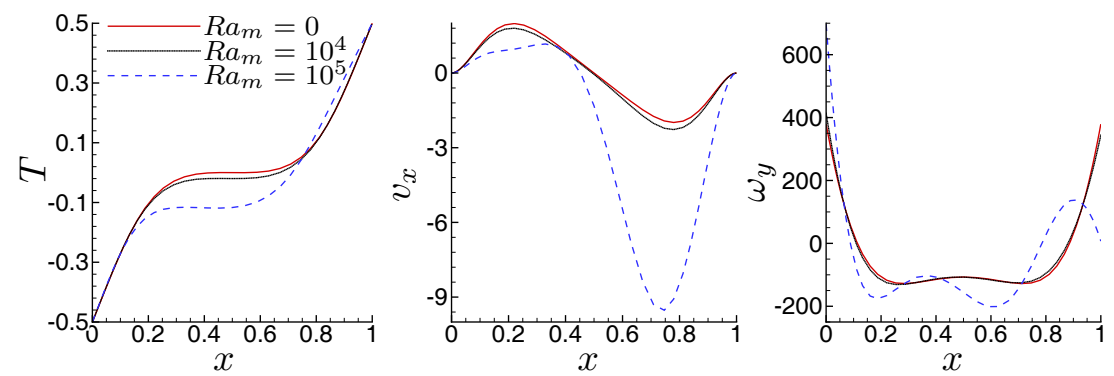

Figure 3: Temperature (left), velocity (centre) and vorticity (right) profiles through the centre of enclosure at $y=0.5$ and $z=0.5$ for $R a=10^{4}$.
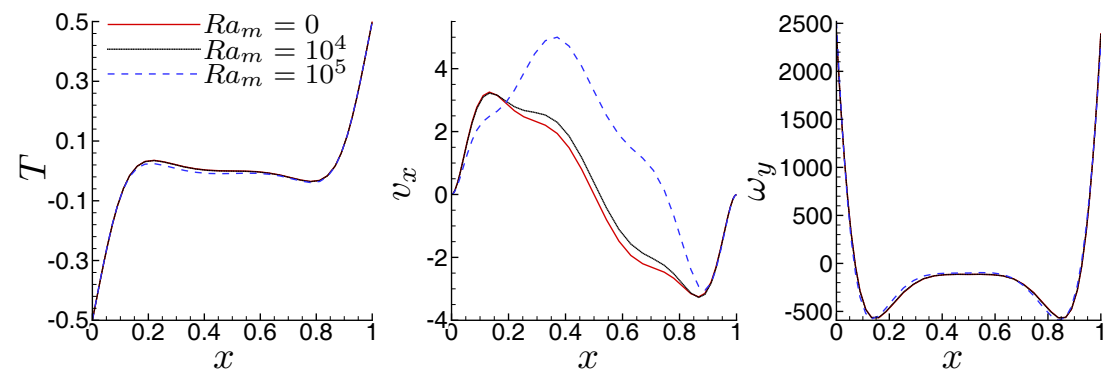

Figure 4: Temperature (left), velocity (centre) and vorticity (right) profiles through the centre of enclosure at $y=0.5$ and $z=0.5$ for $R a=10^{5}$.
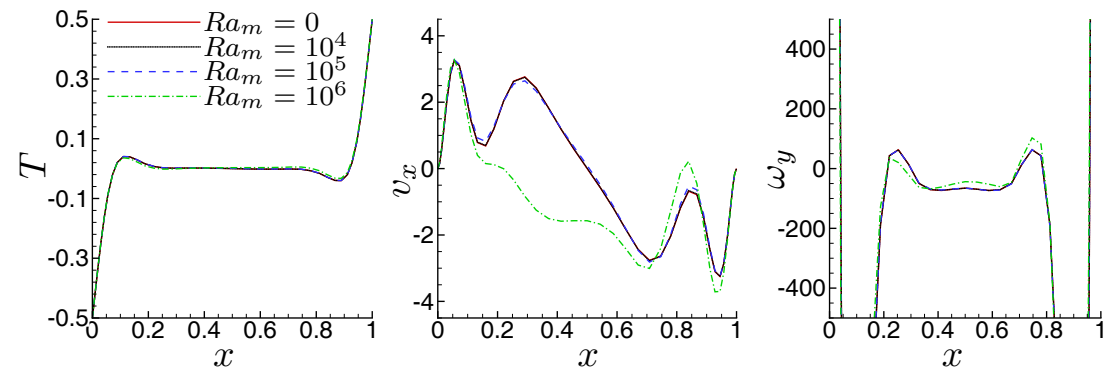

Figure 5: Temperature (left), velocity (centre) and vorticity (right) profiles through the centre of enclosure at $y=0.5$ and $z=0.5$ for $R a=10^{6}$.

We can conclude that the largest differences in the flow field are evident in the velocity field. The temperature field is less affected by the magnetic field. For magnetic Rayleigh numbers less or equal to the flow Rayleigh number $\left(R a_{m} \leq\right.$ $R a$ ) the effects of the magnetic field on the flow field are small, almost negligible. However, for $R a_{m}>R a$ the effects become noticeable. The increase of the 

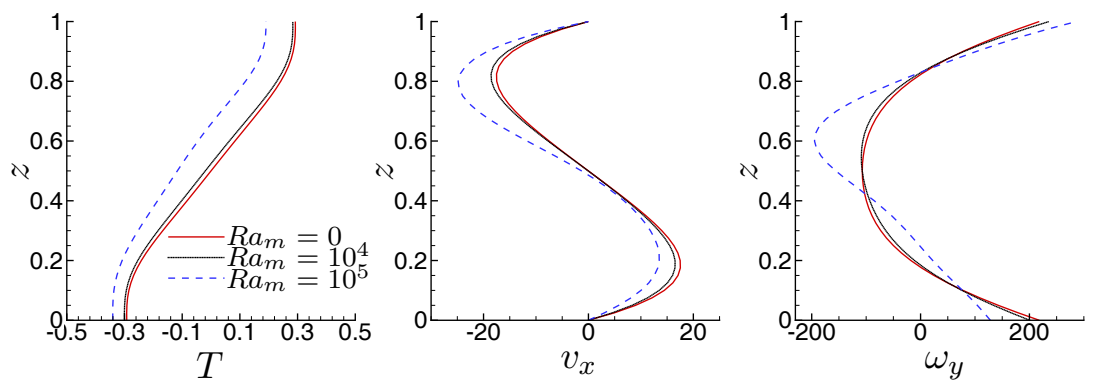

Figure 6: Temperature (left), velocity (centre) and vorticity (right) profiles through the centre of enclosure at $x=0.5$ and $y=0.5$ for $R a=10^{4}$.
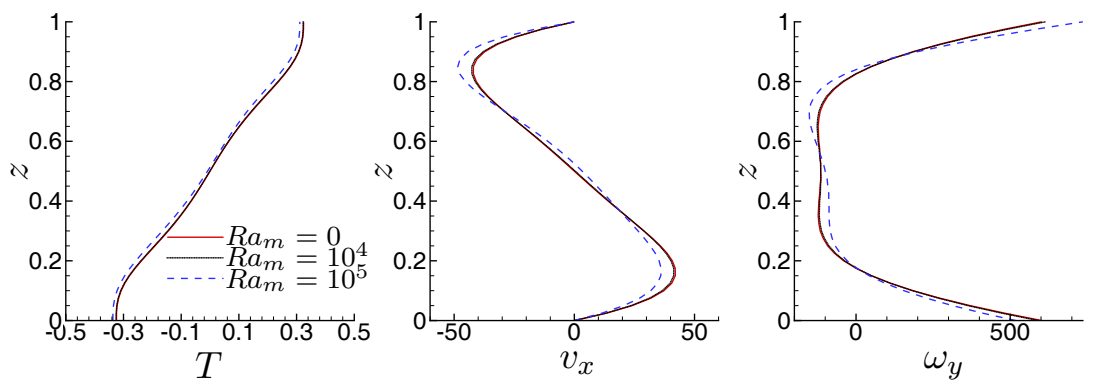

Figure 7: Temperature (left), velocity (centre) and vorticity (right) profiles through the centre of enclosure at $x=0.5$ and $y=0.5$ for $R a=10^{5}$.
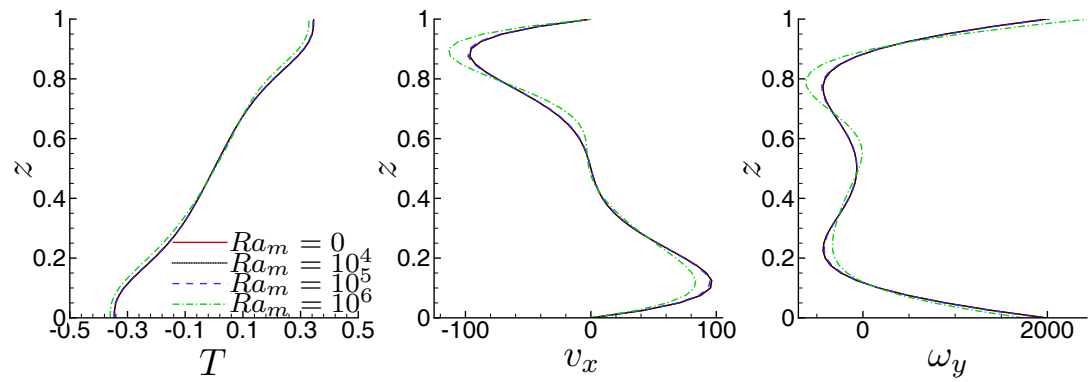

Figure 8: Temperature (left), velocity (centre) and vorticity (right) profiles through the centre of enclosure at $x=0.5$ and $y=0.5$ for $R a=10^{6}$.

magnetic Rayleigh number causes additional body force and contributes to the generation of instabilities in the flow. In the case of $R a=10^{5}$ and $R a_{m}=10^{6}$ the flow was found to be unsteady. The largest effect caused by the magnetic field can be seen in the $v_{x}(x)$ profile. This is due to the fact, that the velocities in the $(x, y, 0.5)$ plane are very small. In the $v_{x}(z)$ profile the velocities are larger and the relative difference between simulations with and without the magnetic field 
smaller. Looking at $T(x)$ profiles, we observe that the magnetic field causes a drop in temperature of the ferrofluid close to the cold wall and a rise close to the hot wall.

\section{Conclusions}

The BEM was applied to viscous flow of ferrofluids in a natural convection problem under the influence of a homogenous external magnetic field. We derived the boundary integral form of the vorticity transport equation, which includes a body force terms, due to the properties of ferrofluids and the external magnetic field.

Through the use of vector algebra and Gauss clause the calculation of derivatives of the body force term has been avoided. Thus the body force term yields a boundary integral term with the fundamental solution as the kernel and a domain integral term, where the gradient of the fundamental solution is the kernel.

The derived numerical model has been used to study the effect of a homogenous external magnetic field on the natural convection of ferrofluids. We established that the effects are negligible for magnetic Rayleigh numbers less or equal to the flow Rayleigh number $\left(R a_{m} \leq R a\right)$. On the other hand, for $R a_{m}>R a$ the magnetic body force changes the flow field significantly and contributes to the instability of the flow.

\section{References}

[1] B. M. Berkovsky, V. F. Medvedev, and M. S. Krakov. Magnetic Fluids Engineering Applications. Oxford Science Publications, 1993.

[2] R. Ganguly, S. Sen, and I. K. Puri. Heat transfer augmentation using a magnetic fluid under the influence of a line dipole. Journal of Magnetism and Magnetic Materials, 271:63-73, 2004.

[3] R. Ganguly, S. Sen, and I. K. Puri. Thermomagnetic convection in a square enclosure using a line dipole. Phys. Fluids, 16:2228-2236, 2004.

[4] R.Y. Hong, Z.Q. Ren, Y.P. Han, H.Z. Li, Y. Zheng, and J. Ding. Rheological properties of water-based $\mathrm{Fe}_{3} \mathrm{O}_{4}$ ferrofluids. Chemical Engineering Science, 62:5912-5924, 2007.

[5] M. Lajvardi, J. Moghimi-Rad, I. Hadi, A. Gavili, T.D. Isfahani, F. Zabihi, and J. Sabbaghzadeh. Experimental investigation for enhanced ferrofluid heat transfer under magnetic field effect. Journal of Magnetism and Magnetic Materials, 322:3508-3513, 2010.

[6] A. Mukhopadhyay, R. Ganguly, S. Sen, and I. K. Puri. A scaling analysis to characterize thermomagnetic convection. International Journal of Heat and Mass Transfer, 48:3485-3492, 2005.

[7] H. W. Müller and A. Engel. Dissipation in ferrofluids: Mesoscopic versus hydrodynamic theory. Phys. Rev. E, 60:7001-7009, Dec 1999. 
[8] C. C. Paige and M. A. Saunders. LSQR: An algorithm for sparse linear equations and sparse least squares. ACM Transactions on Mathematical Software, 8:43-71, 1982.

[9] J. Ravnik, L. Škerget, and Z. Žunič. Velocity-vorticity formulation for 3D natural convection in an inclined enclosure by BEM. Int. J. Heat Mass Transfer, 51:4517-4527, 2008.

[10] J. Ravnik, L. Škerget, and Z. Žunič. Combined single domain and subdomain BEM for 3D laminar viscous flow. Eng. Anal. Bound. Elem., 33:420-424, 2009.

[11] L. Škerget, M. Hriberšek, and Z. Žunič. Natural convection flows in complex cavities by BEM. Int. J. Num. Meth. Heat \& Fluid Fl., 13:720-735, 2003.

[12] P. Škerget and A. Alujevič. The solution of the navier-stokes equations in terms of vorticity-velocity variables by the boundary element method. Gesellschaft angewandte Mathematik und Mechanik, Jahrestagung, Goettingen West Germany Zeitschrift Flugwissenschaften, 65:245, 1985.

[13] T. Tynjälä, A. Hajiloo, W. Polashenski Jr., and P. Zamankhan. Magnetodissipation in ferrofluids. Journal of Magnetism and Magnetic Materials, 252:123-125, 2002. 